\title{
Increased attentional load moves the left to the right
}

\author{
Mario Bonato ${ }^{1,2} *$ and Simone Cutini $i^{3,4}$
}

${ }^{1}$ Department of Experimental Psychology, Ghent University

${ }^{2}$ Department of General Psychology, University of Padova

${ }^{3}$ Department of Developmental Psychology, University of Padova

${ }^{4}$ Center for Cognitive Neurosciences, University of Padova

Running head: Load-dependent contralesional mislocalizations

* Corresponding author:

Dr. Mario Bonato,

Department of Experimental Psychology, Ghent University,

Henri Dunantlaan 2, B-9000 Ghent, Belgium

e-mail: mario.bonato@ugent.be 


\begin{abstract}
Introduction

Unilateral brain damage can heterogeneously alter spatial processing. Very often brain-lesioned patients fail to report (neglect) items appearing within the contralesional space. Much less often patients mislocalize items' spatial position. We investigated whether a top-down attentional load manipulation (dual-tasking), known to result in contralesional omissions even in apparently unimpaired cases, might also induce spatial mislocalizations.

Method

Nine right-hemisphere damaged patients performed three computer-based tasks encompassing different levels of attentional load. The side of appearance of visual targets had to be reported either in isolation or while processing additional information (visual or auditory dual-task). Spatial mislocalizations (from the contralesional hemispace towards the ipsilesional -unaffected- one) were then contrasted with omissions both within and across tasks, at individual as well as at group level.

Results

The representation of ipsilesional targets was accurate and not affected by dual-tasking requirements. Contralesional targets were instead often omitted and, under dual-task conditions, also mislocalized by four patients. Three cases reported a significant number of left targets as appearing on the right (alloesthesia). Two of these patients perceived more targets (albeit to a wrong spatial location) under dual- than under single-task load.

In a fourth patient, increased visual load resulted in synchiria, the (mis)perception of single, contralesional targets as being two (one on each side).

Conclusions

When the neural circuitry subtending spatial processing is damaged, an increase in task load can lead to either a disregard or a bias in the processing of contralesional hemispace. The spatial bias subtending mislocalizations seems to index a more severe deficit than neglect, as if contralesional space would be completely erased rather than merely ignored.
\end{abstract}

Keywords: spatial attention, awareness, alloesthesia, synchiria, allochiria, attentional load, right hemisphere damage, neglect, extinction. 
A coherent phenomenological experience of the world requires the integrated processing of several independent sources of information. For instance, a veridical representation of objects' positions implies a successful interplay between attention and spatial awareness. Here we study how this interplay can be distorted after damage to the right-hemisphere lateralized neural circuitry subtending spatial processing and representation (Bartolomeo, 2014).

It is well established that right hemisphere lesions can result in a number of disorders affecting the processing of left hemispace. Among these disorders, neglect and extinction are the most often described and the most intensely studied (Bartolomeo, Thiebaut de Schotten \& Chica, 2012; Driver \& Vuilleumier, 2001; Vuilleumier, 2013). Both neglect and extinction affect awareness for the presence of contralesional items. Neglect typically indicates the omission of single items presented in the contralesional hemispace. Extinction occurs when a contralesional target remains unperceived only when a second target is concurrently presented in the ipsilesional space. It reflects the winner-takes-all functioning principle of the parietal lobes, which implements the interplay between orienting of spatial attention and awareness (Driver \& Vuilleumier, 2001).

Several lines of evidence converge in showing that the presence and degree of spatial deficits can dramatically increase when several stimuli have to be concurrently processed. Increased attentional demands can hamper contralesional awareness in two different ways: by enlarging the degree of neglected space (Sarri, Greenwood, Kalra \& Driver, 2009) or, when the eccentricity of stimuli is kept constant, by increasing the proportion of missed targets (Bonato, 2012, for review). Due to this dependency on task demands, neglect can be considered as a continuum rather than a categorical disorder. This implies that several patients might present contralesional disorders only when their attentional system is taxed, which is not the case when responding to classic, paper-andpencil, tests. As a result, neglect often remains undetected by these standard tasks.

The strong relation between attentional load and neglect symptomatology derives from a series of studies with RHD patients, whereby a striking increase in contralesional omissions emerged when patients were asked to perform an additional task, either visual or auditory, while monitoring visual space (Bonato, Priftis, Marenzi, Umiltà \& Zorzi, 2010; 2012). Both concurrent tasks similarly hampered patients' awareness for the presence of contralesional targets, irrespective of their different characteristics (Bonato, Priftis, Umiltà \& Zorzi, 2013). This finding was interpreted by assuming the presence of limited and depletable supramodal attentional resources. After brain damage, contralesional awareness would be present only when attention is fully allocated to spatial monitoring, as in the case of clinical tests and of the easy, single-task, condition (see Bonato, 2012, for review; and Marini, Chelazzi \& Maravita, 2013 for a study with healthy participants). Our approach integrates two lines of evidence about load effects on spatial processing. The first one concerns the detrimental effect of increased visual load (mainly at fixation) upon the detection of peripheral stimuli (Ball, Owsley, Sloane, Roenker, \& Bruni, 1993; Bellgrove, Newman, Vance, \& Mattingley, 2013; Buxbaum, Dawson, \& Linsley, 2012; Dawson, Buxbaum, \& Rizzo, 2008; Russell, Husain, \& Malhotra, 2004; Peers, Cusack and Duncan, 2006; Russell, Malhotra, Deidda, \& Husain, 2013; van Kessel, van Nes, Geurts, Brouwer, Fasotti, 2013; Vuilleumier et al., 2008). The second one derives from the coupling between visuospatial and non-spatial tasks (e.g., cancellation under working-memory load, see Robertson \& Frasca, 1992), as well as from studies showing that deficits of contralesional awareness are exacerbated by multimodal contexts (see Jacobs, Brozzoli, Hadj-Bouziane, Meunier, Farnè, 2012, for review).

The present study will capitalize on the remarkable sensitivity of the above described paradigm. We decided to test whether the same bottom-up, dual-task, manipulation which successfully uncovered subtle deficits in spatial awareness, might interfere with the processes allowing an accurate representation of the surrounding space. Accordingly, our first aim will be to investigate whether increased load can unveil mislocalizations. Within the same task, mislocalizations will be next related to the presence of omissions. By looking at omission we will address whether omissions imply the absence of contralesional hemispace coding or whether, in contrast, in omissions contralesional targets can be partly -although unconsciously- processed. To 
our knowledge, this is the first study implementing a dual-task manipulation to reveal mislocalizations.

Among spatial disorders characterizing RHD, spatial mislocalizations are much less frequently described and less understood than omissions. Besides being more difficult to be elicited, mislocalizations are somehow conceptually difficult to envision because, in normal functioning, the perception of an object can hardly be dissociated from the perception of its veridical spatial position. There are, however, some exceptions: when attention is diverted or heavily engaged and when stimuli are peripheral (Treisman \& Schmidt, 1982) even healthy participants can show deficits in feature processing. For instance, under attentional load, the integration (binding) between objects' physical characteristics and its spatial position might fail (Treisman, 1996; 1998) resulting in misattributing a characteristic (e.g., the colour) of one object to another object, thereby causing a sort of transposition (the moving of one characteristic to another location in space). Several phenomena, including the systematic presence of illusory conjunctions within the contralesional hemispace in neglect, strongly support the claim that spatial attention is required for spatial features integration (see Robertson, 2003, for review). Patients with deficits in orienting spatial attention towards the contralesional hemispace provide a unique opportunity to study the determinants of mislocalizations in relation to awareness deficits, also because the performance in the ipsilesional hemispace offers an individually-calibrated baseline.

The two mislocalization disorders we will investigate here are alloesthesia and synchiria. In alloesthesia a single, contralesional stimulus is experienced as ipsilesional (Ortigue, Jabaudon, Landis, Michel, Maravita \& Blanke, 2005) whereas in synchiria a single contralesional stimulus is reported as appearing on both sides (Medina \& Rapp, 2008). Thus, synchiria resembles an alloesthesia-like pattern in the presence of a spared perception of the contralesional stimulus. Both alloesthesia and synchiria have a spatial origin (and not a visual one). As a consequence, in analogy with other spatial disorders like neglect and extinction, alloesthesia can be induced both within and across different sensory modalities (Brozzoli, Demattè, Pavani, Frassinetti, \& Farnè, 2006; Ortigue et al., 2005; Pavani et al., 2004) and synchiria can be modulated by head- and trunk-defined spatial frames of reference (Medina \& Rapp, 2008). Moreover, both synchiria and alloesthesia have been investigated in multiple sensory modalities. Neppi-Modona (1999) applied tactile stimulation in a group of right hemisphere damaged patients. His study concluded that, when using a simple localization task, alloesthesic responses are not common: out of thirty RHD patients, alloesthesic responses were about $3 \%$ for left-sided stimuli and about $1.3 \%$ for right-sided stimuli. Moreover, no lateralized response bias emerged out of a number of catch trials (no stimulation) as high as 2400 . Also sounds mislocalization has been described (Brozzoli et al., 2006). Spatial distortions in the perceived localization of auditory stimuli can be, at least partly, due to motor neglect and body schema distortions, in particular when patients are asked to point to the perceived sound position (Brozzoli et al., 2006; Karnath, 2015). Mislocalizations can emerge under heterogeneous formats and concurrently with additional deficits. For instance, some previous studies on alloesthesia also reported transpositions from the ipsilesional to the contralesional hemispace, often occurring concurrently with palinopsia (Jacobs, 1980; Ardila, Botero, \& Gomez, 1987).

In summary, here we investigated whether a dual-tasking procedure could unveil mislocalizations in a small group of neurological patients which were potentially affected by impaired spatial coding due to a neural damage in the hemisphere specialized for spatial processing.

\section{Method}

Sample

We performed a re-analysis of a dataset already available (cases selected from Bonato et al., 2010; 2012), with the addition of two new patients (see Bonato et al., 2013). The comparisons here performed are new and substantially different from our previous studies because for the first time the different types of wrong responses to contralesional stimuli were analyzed. 
All data had been collected while patients were admitted to the Rehabilitation Center of Conselve (Padova). Inclusion criteria were the presence of a single right hemisphere lesion and having performed, with a target duration of 50ms, the computerized single/dual-task described in Bonato et al., (2012). With short target duration, task sensitivity is maximal because a bottom-up attentional engagement is added to top-down processes determined by dual-tasking (Bonato, 2015). The final sample encompassed nine patients (four males) aged 40-85 (mean 67 years) (see Table 1), tested on average 96 days (range 24-476) after lesion onset.

Neuropsychological testing

All of the patients had been tested with a comprehensive neuropsychological screening (ENB, Mondini, Mapelli, Vestri, \& Bisiacchi, 2003) and with a battery for neglect (Behavioural Inattention Test, BIT; Wilson, Cockburn, \& Halligan, 1987). The ENB includes several subtests assessing memory, executive functions and visuospatial processing. It includes a drawing copying subtest in which the patient is asked to copy a drawing of a house in a blank space below the example house. The BIT consists of three cancellation tasks (Lines, Letters, Stars), four copying, and three drawing from memory tasks, plus a line bisection subtest.

--- Table 1 about here ---

\section{Computer-based tasks}

The computer-based tasks (see figure 1) encompassed one single-task and two dual-tasks. All stimuli were identical across tasks, whereas instructions were task-specific. In all tasks, after a central fixation cross (1000 ms) a visual target (a black circle -diameter 8mm-) appeared for $50 \mathrm{~ms}$ on the left, on the right, or bilaterally (eccentricity $135 \mathrm{~mm}$ ). Concurrently, a letter (a, b, v or z) was shown at fixation and a number $(1,2,8$ or 9$)$ was presented auditorily. In the single task (ST) patients only had to report the spatial position of target(s) (i.e., "right", "left", or "both" sides), and to ignore both the visual letter and the auditory number. In the dual-tasks, before reporting the position of the target(s), patients had to: i) read the letter (visual dual-task, VDT) or ii) count forward by two twice starting from the heard number (auditory dual-task, ADT). The experimenter (MB) recorded the responses to the target(s) and to the concurrent task (when present). Performance was therefore recorded in terms of accuracy only and reaction times were not collected. A noisy screenshot was then presented until response.

In five patients (Cases 2, 4, 5, 7 \& 8) taken from Bonato et al., (2012) also data obtained with a longer target duration (between 500 and $600 \mathrm{~ms}$ ) were available.

After having explained the task, the experimenter pointed to the expected position of appearance of targets on the screen to ensure that patients correctly named left/right/bilateral sides. All patients were successful in naming/pointing towards the spatial position indicated by the experimenter. Note that healthy, elderly participants perform at ceiling on these three tasks (Bonato et al., 2010; Bonato, 2015). 


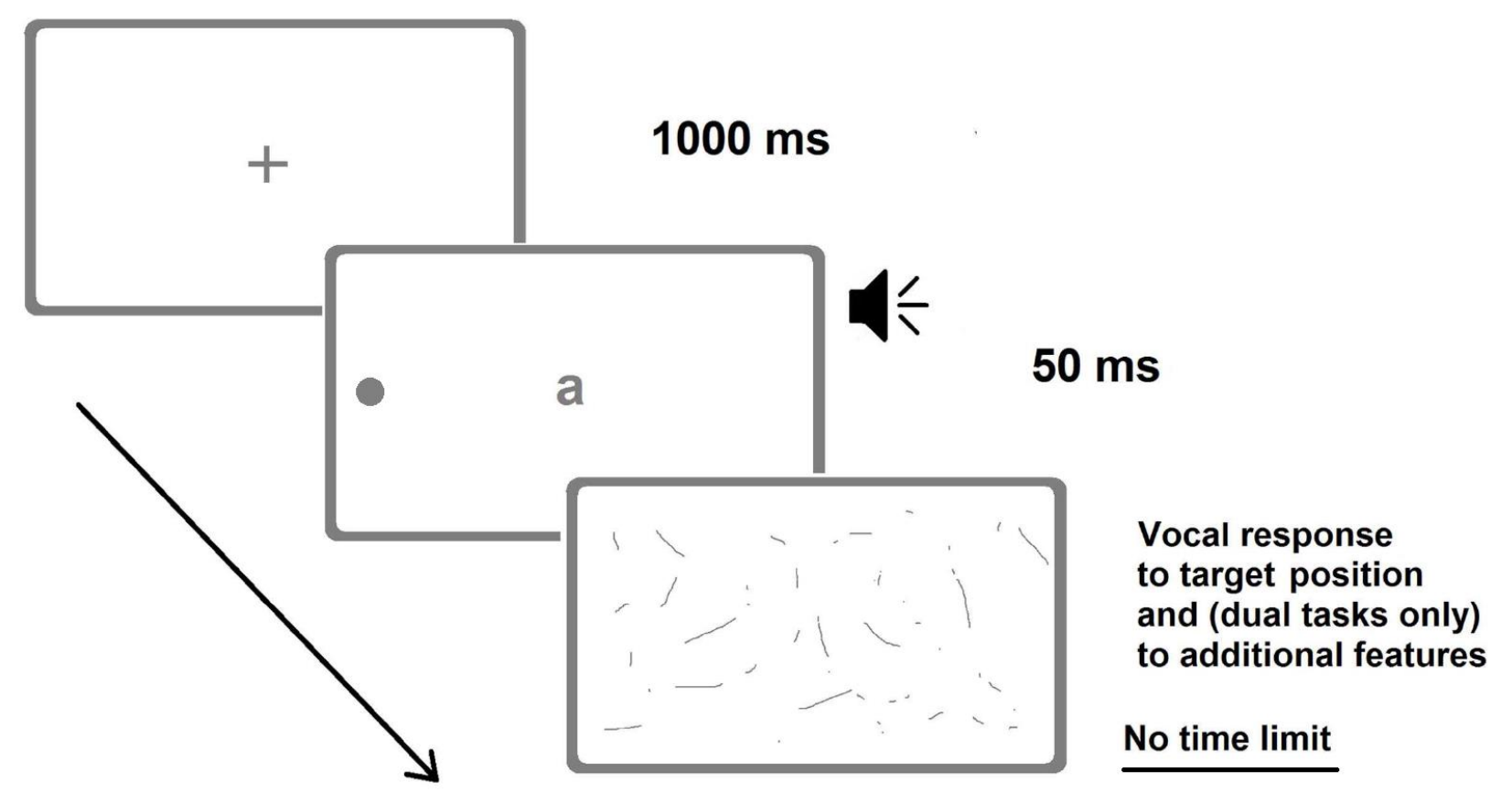

Figure 1

A schematic representation of the task (adapted from Bonato, 2012 \& 2015) is shown.

Eye movements were recorded with a camcorder focused on patients' eyes. Task order was counterbalanced across participants. Each of the three tasks encompassed two blocks of 48 trials, resulting in a total of 288 trials for each patient. Trials contaminated by eye movements $(<2 \%)$ and with a wrong response to the secondary task $(\approx 20 \%$ in the VDT, $\approx 2 \%$ in the ADT) were discarded. Case 6 was administered with a previous task version whereby auditory numbers were presented in the ADT condition only.

Double simultaneous stimulation

In order to provide a baseline measurement of visual mislocalizations and omissions in a standard neurological test patients were asked detect brief, unilateral or bilateral, movements of the index finger(s) of the examiner (DSS: Double Simultaneous Stimulation). DSS is one of the most sensitive clinical tasks for quantifying contralesional spatial deficits (Maravita, Posteraro, Husain, Vuilleumier, Schwartz \& Driver, 2007), and broadly resembles the single task condition of our computer-based experiments. Each patient had to detect 84 visually-presented stimuli (left, right or bilateral), half for the upper and half for the lower visual field, in a fixed random order (Bisiach, Cappa, \& Vallar, 1983).

\section{Results}

Behavioural Inattention Test

Average score at the BIT was 134 out of 146, with two patients out of nine showing a performance below the cut-off of 130/146 (Case 4: 124 and Case 8: 129, see Table 1). No patient had neglect according to the omissions at the cancellation tasks (lines, letters and stars cancellation $93 \%$ left vs. $94 \%$ right targets cancelled, all comparisons $n s$ ).

Computer-based tasks

Patients' responses at the computer-based tasks are shown in Table 2. A group analysis was first performed to compare correct responses across positions and tasks. Individual accuracies were analyzed by means of a 3 (Target Position: left vs. bilateral vs. right) X 3 (Task: ST vs. VDT vs. ADT) ANOVA. All effects were significant: the main effect of Target Position, $F(2,16)=29.1, p<$ .001 , the main effect of Task, $F(2,16)=7.6, p<.01$, and the Target Position $\mathrm{X}$ Task interaction, $F(4,31)=3.6, p<.05$. The interaction was seemingly due to the absence of differences between 
tasks (and, more in general, to the limited number of errors) occurring for right-sided targets only (mean accuracy $=97.2 \%$ in ST, $93.4 \%$ in VDT, and 94.7\% in ADT). To confirm this

interpretation, an ANOVA assessing task effects separately for each target side was performed. The main effect of Task was significant for left, $F(2,16)=4.7, p<.05$ and bilateral targets, $F(2,16)=$ $7.8, p<.01$, but not for right ones $F(2,16)=1.5, p=.25$. Responses to ipsilesional targets were overall accurate and therefore unaffected by the load manipulation also at individual level (all $p s>$ $.05)$, see Table 2, right column. Responses to bilateral targets were more accurate in the ST (47\%) than in the VDT, $(18 \%) t(8)=2.7, p<.05$ and in the ADT, $(17 \%) t(8)=2.9, p<.05$. Also for left targets, accuracy was higher in the ST (56\%) than in both the VDT $(25 \%), t(8)=2.96, p<.05$ and the ADT (33\%), $t(8)=1.96, p<.05$ (one-tailed), as already documented in Bonato et al., (2013). We then tried to understand whether such decrease in accuracy was only due to increased omissions or whether it was, at least in part, due to the appearance of wrong responses for contralesional targets under dual task load(see later and Fig. 3).

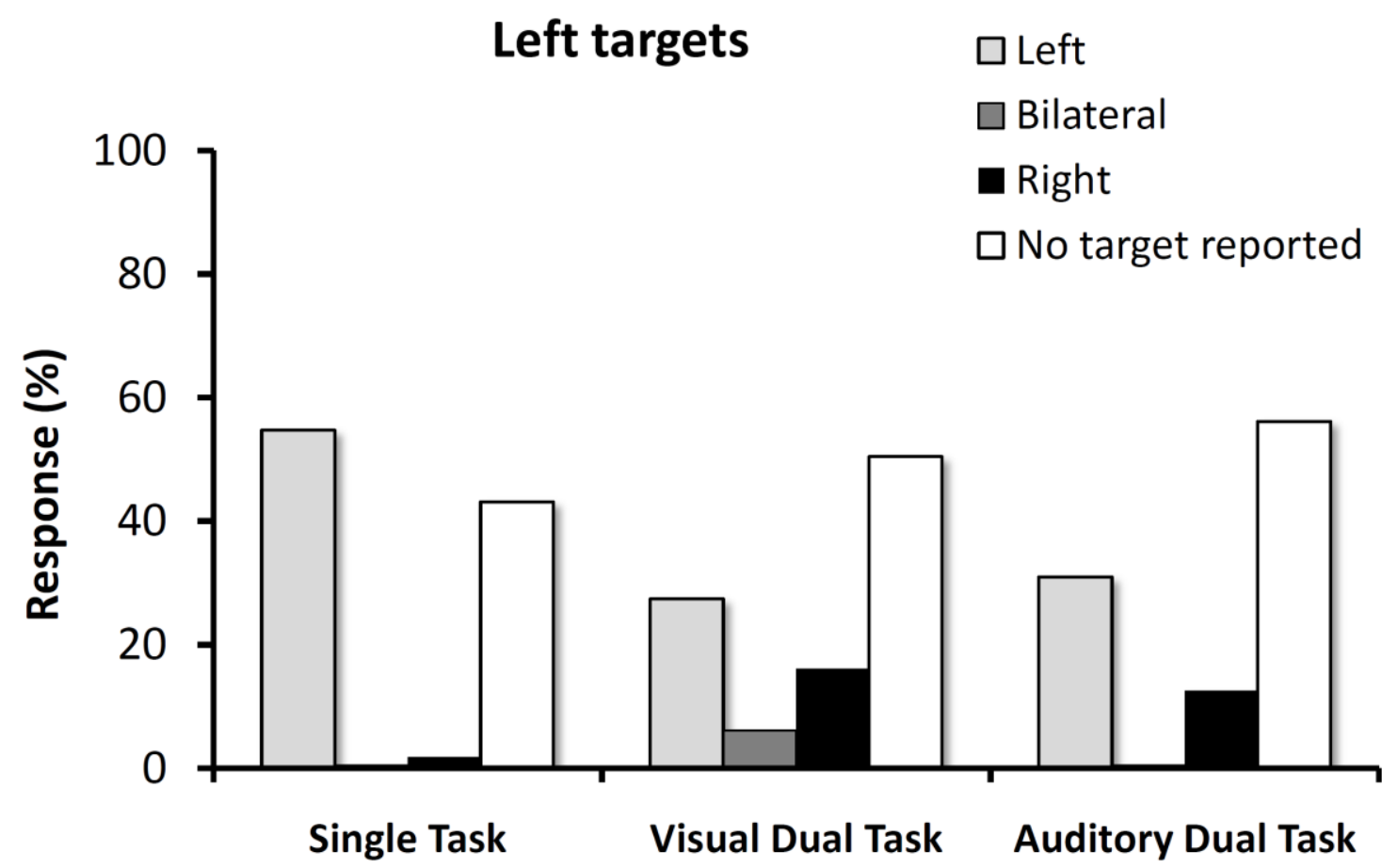

Figure 2

Average (group) responses to left targets are reported, separately for each task. Alloesthesic responses are represented in black and synchiric responses in dark grey. White and light grey bars indicate omissions and correct responses, respectively.

After having ensured that performance accuracy was affected by attentional load only in the contralesional hemispace, we distinguished omissions from mislocalizations. We considered as omissions both the trials where patients responded that they did not perceive any target and those in which no answer occurred.

According to these criteria omissions for left targets were $43 \%$ in the ST; $52 \%$ in the VDT; and $55 \%$ in the ADT, see Figure 2). Compared to the high number of omissions, mislocalizations for left targets (i.e., "right" or "both" responses) were a negligible number (1.8\%) in ST.

In contrast, under dual-tasking, some mislocalizations emerged $(23.5 \%$ in VDT and $12.6 \%$ in ADT). Their number highly varied across patients (from $0 \%$ to 67\%) and their presence across tasks was then analysed at an individual level. We only considered for individual analysis patients who misplaced target position in more than five trials in at least one condition. Four patients showed 
mislocalizations, three of whom showed alloesthesia and one showed synchiria. These cases are individually presented below.

$$
\text { --- Table } 2 \text { about here --- }
$$

Case 2 showed a significant increase in the number of left targets perceived as appearing on the right (i.e. visual alloesthesia) from $0 \%(\mathrm{ST})$ to $67 \%(\mathrm{VDT}), \chi^{2}(1, N=37)=21.33, p<.001$, (Fisher's Exact Test, here and later). Performance in the ADT (10\% of alloesthesic perceptions), was not significantly different from ST, $\chi^{2}(1, N=38)=1.9, p=.28$.

In Case 4 the number of left targets perceived as appearing on the right significantly increased from $3.1 \%(\mathrm{ST})$ to $65 \%$ in the VDT, $\chi^{2}(1, N=16)=9.9, p<.05$, and to $34 \%$ in the ADT, $\chi^{2}(1, N=14)$ $=8.56, p<.05$. The difference between VDT and ADT was not significant, $\chi^{2}(1, N=24)=1$, ns. Case 8 showed $28 \%$ of alloesthesic responses in the ADT, significantly more than in the VDT, $\chi^{2}$ $(1, N=12)=7.2, p<.05$ but not than in the ST. The difference between the ST (9\%) and the VDT $(3.1 \%)$ was not significant, either, $\chi^{2}(1, N=6)=3, p=.4$.

We then tested whether alloesthesia under dual-task conditions was accompanied by a change in the number of omissions with respect to the ST condition. Indeed, for Cases 2 and 4, that was the case. Alloesthesia under dual tasking occurred with a concurrent, significant, reduction of contralesional omissions with respect to ST (all $p$ s $<.05$, see Table 2 ). In contrast, the remaining patients showed more omissions in dual-tasking than in the ST.

One patient (Case 1) showed a different pattern of mislocalizations, called synchiria and consisting in "bilateral" responses to left targets. In her case synchiric responses emerged selectively in the VDT (54\%), with a significant difference with respect to the ST $(4 \%), \chi^{2}(1, N=47)=15.4, p<$ .001 and to $\operatorname{ADT}(3.7 \%), \chi^{2}(1, N=50)=17.2, p<.001$. To assess whether synchiria occurred selectively for contralesional targets and to exclude the impact of a potential response stereotypy, the same analysis was performed after having subtracted the percentage of "bilateral" response to ipsilesional targets ( $0 \%$ in the ST/ADT; $16 \%$ in VDT). The difference between the VDT and both the ST and the ADT remained significant $\chi^{2}(1, N=43)=11.09, p<.001$ and $\chi^{2}(1, N=46)=$ $12.49, p<.001$ after the above mentioned correction. Case 1 did not show alloesthesia in ST (0\%), VDT $(4 \%)$ nor in ADT (0\%). As opposed to the other patients she did not show neither omissions nor extinction under load.

In summary: a significant number of mislocalizations occurred in four patients. Three patients (Cases 2, 4 and 8) showed alloesthesia (reported left targets as right) and one (Case 1) synchiria (reported left targets as appearing bilaterally). These mislocalizations were space-selective (emerged for left targets) and load-selective (emerged under dual-task conditions only; See Figure 3 and Table 2)*. 

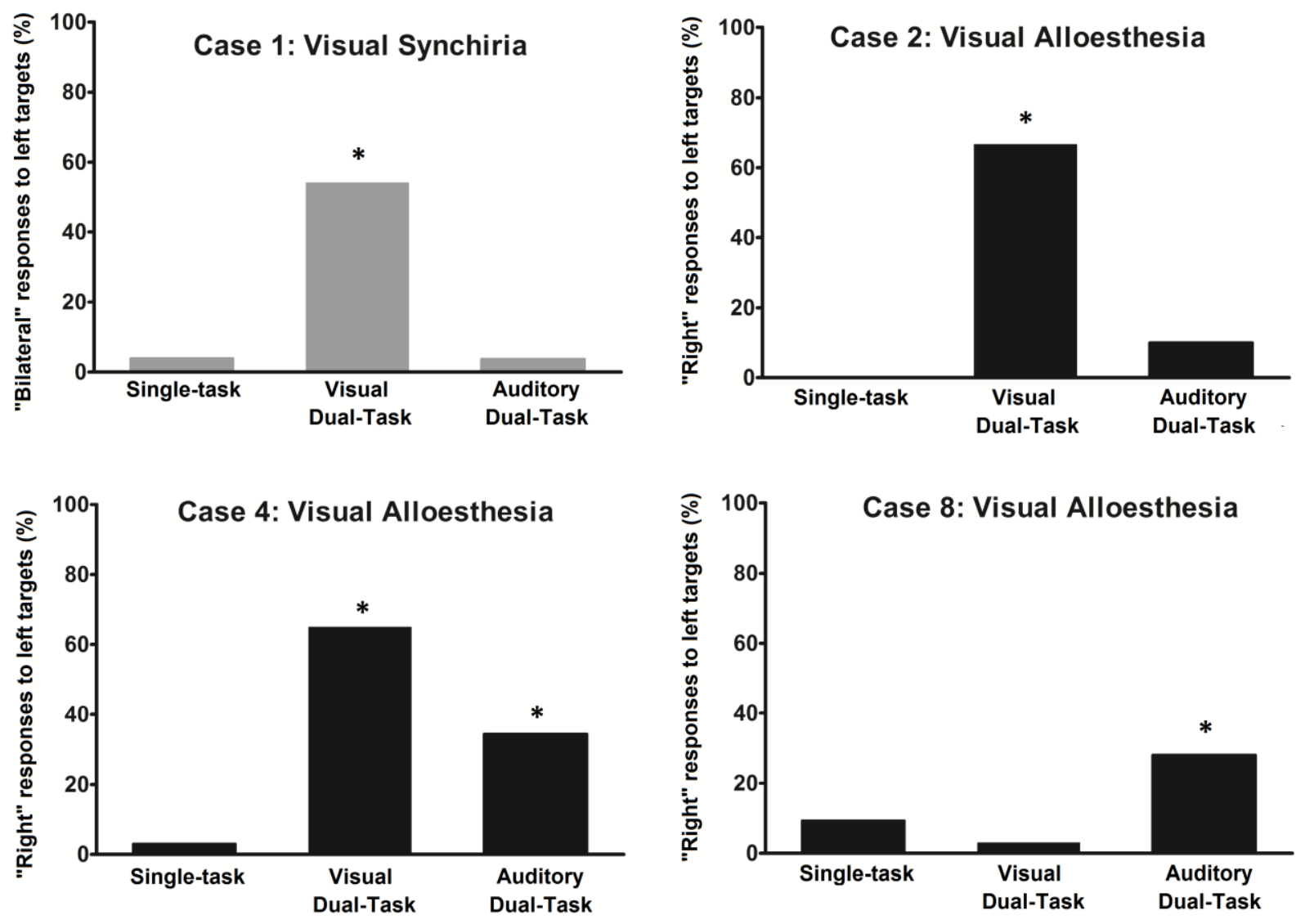

Figure 3

Wrong responses for contralesional items are reported, separately for all patients presenting a significant rate of mislocalizations and for each task. Cases 2, 4 and 8 presented alloesthesia (black) whereas Case 1 presented synchiria (grey).

In Cases 2 and 8, two patients with alloesthesia, the "long duration" version gave rise to a negligible number of alloesthesic responses (2.1\% and 3.1\%, for Cases 2 and 8 , respectively). The same target duration successfully induced severe extinction (>95\%) under dual-tasks across all tasks (Case 2) or under dual-tasks only (Case 8). In contrast, patients' detection of contralesional single targets was relatively spared (omissions $<20 \%$ ). It is worth reminding that, with the shorter target duration $(50 \mathrm{~ms})$, omissions for contralesional single targets were, under single task, as high as $54.8 \%$ (Case 2) and 90.6\% (Case 8) and that alloesthesia emerged under dual-tasks only.

Double simultaneous stimulation

No patient presented a significant number of alloesthesic/synchiric responses (all $p$ s vs. $0 \mathrm{~ns}$ ) at the DSS.

Drawing/copying tests

Patients' performance was also analysed in those paper-and-pencil tasks which might have revealed spatial mislocalizations. Across all the drawing-from-memory (five) and copying (five) tests administered to each patient (BIT + ENB) two examples of allochiria emerged in the ENB copy subtest (see Figure 4). Allochiria is a physical transposition from the contralesional to the ipsilesional space (Halligan, Marshall \& Wade, 1992; Lepore, Conson, Grossi, \& Trojano, 2003). It occurred for Cases 3 and 5, two patients who did not present any mislocalization at the computerized tasks. 


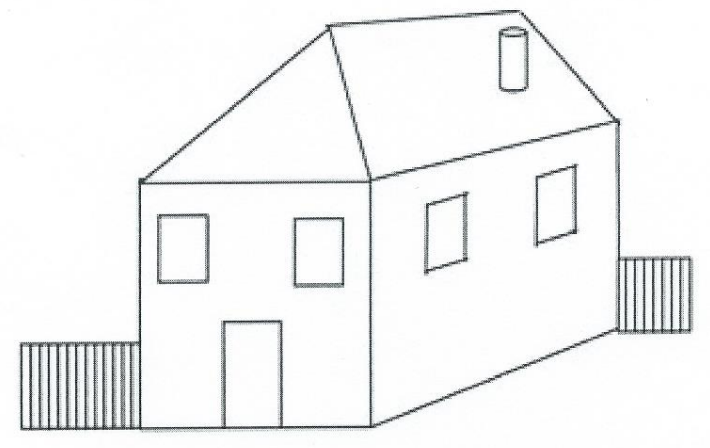

A

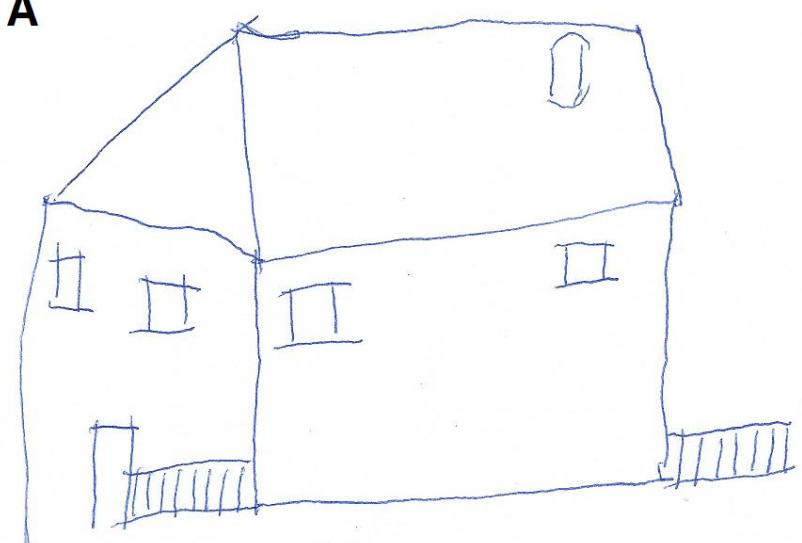

B

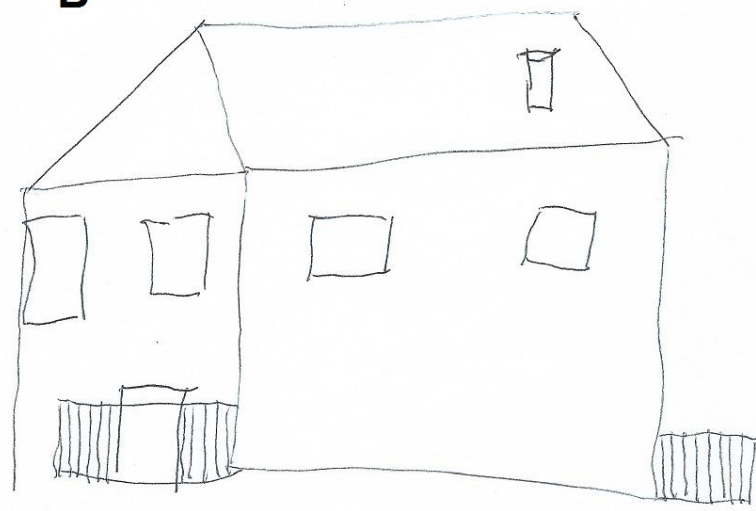

Figure 4

The figure shows in its upper part the item (house) to be copied as part of the ENB screening. In the lower part of the figure the allochiric performance of two patients is visible. Case 5 transposed the fence from the left to the right (Fig. 4, panel A) whereas Case 3 symmetrically drew two fences, one for each side of the door showing a sort of object(door) -based synchiria (Fig. 4, panel B).

\section{Discussion}

Here we have shown that, after right hemisphere damage, increased attentional load can not only diminish the processing of contralesional information but also distort its representation. Under dualtask conditions selective mislocalizations emerged, whereby contralesional targets were reported by some patients to appear in the ipsilesional hemispace or bilaterally. We used a computer-based task requiring patients to monitor visual space for target appearance either in isolation or while being asked to also report an additional stimulus feature. The dual-task manipulation differently affected contralesional spatial processing. It led four patients to misreport targets' spatial position, whereas it led to unawareness in the remaining five patients. In contrast with the impairments found for contralesional hemispace, performance for ipsilesional targets was almost errorless and unaffected by task load across all patients.

When the neural circuitry subtending spatial processing is damaged, the veridical representation of spatial location seems to be strongly dependent on the availability of unspecific attentional resources. Indeed, mislocalizations emerged following purely top-down manipulations rather than the manipulation of perceptual factors alone (e.g., reduced presentation time). Concurrent task performance plausibly reduced the availability of attentional resources which otherwise would have sufficed to effectively process the contralesional hemispace under single-task conditions ( Bonato et al., 2010). A resource-based explanation can easily account for the good performance in the -less demanding- DSS testing/single-task conditions, and it is also in line with the fact that patients presented less severe impairments when targets were presented for a longer duration, customized to the individual level of impairment (Bonato et al., 2012). 
It is unlikely that mislocalizations were in fact a response bias, with patients responding "right" or "bilateral" whenever they did not perceive the target. From a methodological point of view, verbal responses without time-pressure (as opposed to key-presses, which are sensitive to motor neglect) are considered an "unbiased" response index (Bisiach, Ricci, Lualdi, \& Colombo, 1998; see also Neppi-Modona, 1999) and are widely used to calculate the incidence of visual disorders after brain damage (Baier, de Hann, Meller, et al., 2010). From an empirical point of view, this conjecture is at odds with several aspects of the data. Under dual-task conditions, alloesthesic responses still occurred together with residual omissions: an automatic "right" response would not have led to omissions. Moreover, a stereotypic response would be hardly compatible with the good neuropsychological profiles of the patient and it should have emerged also when target duration was longer and under single-task conditions. Finally, it would have hardly dissociated across dual tasks (as found for Cases 1,2, and 8).

On top of that, spatial displacement from contralesional towards ipsilesional hemispace is thought to index genuine distortions of contralesional space (see Pavani, Husain, Làdavas \& Driver, 2004 for auditory and Liu et al., 2011 for tactile sensory modality). The same logic holds for synchiria. Indeed, the fact that Case 1 presented several correct detections of contralesional targets and presented synchiric responses only in one dual-task -and only for left but not for right targetsallows ruling out the presence of a generalized response bias. If this was the case, "bilateral" responses would have emerged under both dual-task conditions and also for single, unilateral, right targets.

The "shift" of left items to the right here described is compatible with the pathological, automatic orienting of spatial attention which, after right-hemisphere damage, is easily triggered towards ipsilesional hemispace (Bonato, Priftis, Marenzi \& Zorzi, 2009).

The binding of the sensory information into a coherent percept occurs through the coding of the position of items in space and by linking objects' physical features with their spatial locations (Cohen \& Rafal, 1991; Robertson, 2003; Treisman, 1996). Since the processing of objects' shape and location occurs through separate cognitive systems and neural structures, only when the binding is effective a coherent perception of features in space can emerge (Robertson, 2003).

Present findings support the view that the binding process requires spatial attention resources to successfully occur (Marcel et al, 2004; Treisman, 1996; 1998). Indeed, we showed that under load the representations of items are mislocated FROM a contralesional location -where spatial attention can only in some contexts be allocated as if "no reliable space" would exist there- TOWARDS a location where spatial attention is pathologically attracted.

Present data also suggest that the heterogeneous consequences of increased load across patients might be, at least in part, related to the initial severity of the deficit. When contralesional awareness was already low in the single-task, load increase more easily led to spatial transpositions. This conjecture seems to be confirmed by the data: with the exception of the only non-vascular patient (Case 7) no patient with a "baseline" extinction below 50\% presented alloesthesia. In contrast, three out of four patients with "baseline" omissions above $50 \%$ showed alloesthesia and all patients with alloesthesia also presented an extinction rate $>90 \%$ in the same conditions. In contrast, when fewer omissions were present under single-task conditions, load increase more probably resulted in omissions (rather than in transpositions).

These data do not support the -admittedly tempting- possibility to conceive alloesthesia as an intermediate step in a gradient leading from neglect to extinction. It seems, in contrast, that alloesthesia (under dual task) was only present in patients showing several omissions under ST. In the two patients with severe alloesthesia (i.e., > 50\%) more targets were omitted in the single than in the dual-task condition. It therefore seems as if the same stimuli, which were not consciously perceived under single-task conditions, "re-entered" awareness under load, albeit at a wrong location. Thus, mislocalizing may be worse than omitting. The present findings seem to suggest that alloesthesia might be due to a distortion of the contralesional spatial map, whilst neglect would 
instead be present when a failure in the access to such map (Robertson, 2003) occurs. This interpretation would be consistent with the body of evidence showing that contralesional stimuli which are not consciously perceived can nonetheless influence performance of neglect patients. Supporting evidence comes from priming (Marshall \& Halligan, 1988), from redundant-target effects (Marzi et al., 1996) and from the physiological activation of primary visual areas in the absence of conscious perception (Rees, Wojciulik, Clarke, Husain, Frith \& Driver, 2000). Counterintuitively, alloesthesia might be then somehow considered a spatial impairment more "disruptive" than neglect, rather than the opposite. It seems as if load would not allow creating a representation of contralesional space rather than merely hampering its access as if the "only" space available for representing spatial positions was the ipsilesional one.

In summary, computer-based tasks revealed load-dependent mislocalizations and the presence of a gradient in the severity of unawareness for contralesional hemispace from extinction as less severe deficit, to then go to neglect, and, finally, to alloesthesia.

According to BIT scores, most of the patients had no neglect. It might therefore be interesting to speculate about the pattern of results which should be expected in a clinical sample characterized by severe neglect. These hypothetical patients will no doubt omit many contralesional targets already in the ST. The question then becomes whether they will present alloesthesic responses already in the ST, and whether they will present, under dual-task, more mislocalizations than the group here described. We speculate that, as in the sample we tested, mislocalizations would not affect all patients and synchiria will be uncommon. It seems also plausible to predict, in the most affected patients, a severe alloesthesic pattern under dual-tasking. Finally, the relatively high (16\%) percentage of synchiric responses to ipsilateral trials, as well as its presence in one patient and in one condition only, calls for caution when the potential frequency of presentation of this disorder has to be estimated.A second dissociation was found between synchiria and neglect/extinction, and it is supported by the fact that Case 1 showed synchiria with no hint of neglect and extinction nor alloesthesia, neither at clinical testing nor under computer-based tasks.

The data suggest that spatial processing is multicomponential and that its proper functioning requires the integration of several different processes subtending awareness and spatial localization. As a result of a disruption to the mechanisms subtending spatial processing, a target can be consciously perceived but in a wrong spatial location. This impaired spatial coding in the presence of preserved awareness complements recent evidence suggesting the possibility of preserved spatial coding in the presence of impaired awareness (Treccani, Cubelli, Sellaro, Umiltà \& Della Sala, 2012). Moreover, the fact that targets which were not consciously perceived in single task conditions were mislocated under dual task conceptually replicates the Treccani et al. findings. Patients with alloesthesia under visual load (Cases 2 and 4) might have presented, in the single task, some representation for the presence of contralesional targets which could however not enter awareness.

The dissociation between mislocalizations on the computerized tasks and allochiria on the drawing copying (see Halligan et al., 1992) suggests that, in allochiria, transpositions might be due to the modality of output. The potential presence of directional hypokinesia would then affect motor (allochiria) but not verbal (alloesthesia/synchiria) responses. According to this speculation, alloesthesia would then be a more veridical index of spatial distortions.

Crucially from a clinical perspective, the present results suggest that standard neurological (DSS) approach is not sufficiently sensitive to induce and detect mislocalizational disorders. The ceiling performance found at the DSS resembled the absence of mislocalizations found under computerbased, single-task conditions, suggesting that the brief presentation of stimuli is a necessary but not a sufficient condition for alloesthesia to occur. Moreover this suggests that low-demand tasks, despite being very sensitive for the detection of omissions, are still not sufficiently demanding to induce (nor sensitive to detect) mislocalizations, confirming the above proposed severity continuum between extinction, neglect and alloesthesia. 
Rather obviously, it is not the use of a computer in itself but rather the requirement to concurrently process various stimuli which results in mislocalizations (Bonato \& Deouell, 2013). On top of that, it is possible that some alloesthesic responses might in fact go undetected in the presence of extinction (Medina \& Rapp, 2008).

The degree of initial neglect severity provides a potential explanation as to why in some patients the load manipulation leads to omissions whereas in some others it led to mislocalizations. However, this does not imply that all neglect patients with severe neglect must present mislocalizations under load. In the presence of damage to the circuitry subtending spatial processing, the need to devote attention to several aspects of the stimuli seems be crucial to induce mislocalizations. Evidence by research in the multimodal domain supports this possibility (Ortigue et al., 2005). The patient described by Ortigue et al., (2005) showed alloesthesia for contralesional task-relevant stimuli, either auditory or tactile when task irrelevant, cross modality, distracters (auditory for tactile stimuli and tactile for auditory ones) were presented within the ipsilesional hemispace.

A major limitation of the present study is the absence of lesion mapping due to the unavailability of neuroradiological images for two patients. Even taking into account the scans we had available, the lesions resembled the well-known heterogeneity of the areas potentially leading to neglect. As a consequence of the limited sample and of the heterogeneity of the lesions, any anatomically-based explanation for the presence of these dissociations at individual level would seem unjustified. Another limitation is the presence of a large variability in the degree and characteristics of mislocalizations and omissions. For instance, whereas for Cases 1, 2, and 4 performance was less impaired in ADT than in VDT, for Case 8 the opposite was true.

Even focusing on omissions only, Case 6 was characterized by a larger number of them in the ADT than in the VDT. This could be easily explained by assuming a "novelty effect" due to the auditory presentation (Case 6 underwent the auditory number presentation in the ADT only). This conjecture, however, falls short in explaining the reason why the same patient, under bilateral target presentation, presented an identical rate of omissions (extinction) in both dual tasks. It therefore remains currently unexplained the heterogeneity of the factors leading to omissions and mislocalizations. It is possible that deficits emerged in those conditions which were individually more difficult, but this is, for the moment, only a speculation.

Nonetheless, the current results clearly illustrate the deficits that can arise in the computation of the spatial locations of stimuli and in the integration of position information into a coherent spatial representation.

\section{Appendix}

The definitions of alloesthesia and allochiria remarkably vary across studies (Halligan et al., 1992; Jones, 1907; Lepore et al., 2003; Marcel, Postma, Gillmeister, Cox, Rorden, Nimmo-Smith et al., 2004; Meador, Allen, Adams \& Loring, 1991; Medina \& Rapp, 2008; Obersteiner, 1882; Ortigue et al., 2005; Treccani et al., 2012). We have adopted the term "alloesthesia" (Ortigue et al., 2005) to indicate the mislocalization of contralesional items within the ipsilesional hemispace in those contexts (e.g. the computer-based task we administered) without motor component. We used the term "allochiria" to indicate the occurrence of spatial transpositions from the contralesional to the ipsilesional hemispace only within copying/drawing from memory tasks, that is when the target item had to be "physically" reproduced (Halligan et al., 1992).

Even though, broadly speaking, extinction might also be considered a type of mislocalization (e.g. an alloesthesic phenomenon), we preferred to classify it (together with neglect) as an awareness deficit. 


\section{Acknowledgements}

This study was supported by a FWO Pegasus/Marie Curie Intra European Fellowship within the 7th European Community Framework Program to M.B. S.C. was supported by grant STPD11B8HM from the University of Padova.

$\mathrm{MB}$ is grateful to K. Priftis and to two anonymous reviewers for helpful suggestions on a previous version of this paper; to M. Zorzi, C. Umiltà, P. Bartolomeo and R. Cubelli for useful discussions, and to J-P van Dijck for proofreading.

\section{References}

Ardila, A., Botero, M., \& Gomez, J. (1987). Palinopsia and Visual Allesthesia. International Journal of Neuroscience, 32, 775-782.

Baier, B., de Haan, B., Mueller, N., Thoemke, F., Birklein, F., Dieterich, M., \& Karnath, H.-O. (2010). Anatomical correlate of positive spontaneous visual phenomena: a voxelwise lesion study. Neurology, 74, 218-222.

Ball, K., Owsley, C., Sloane, M.E., Roenker, D.L., \& Bruni, J.R. (1993). Visual attention problems as a predictor of vehicle crashes in older drivers. Investigative Ophthalmology Visual Science, 34, 3110-3123.

Bartolomeo, P. (2014) Attention disorders after right brain damage. Living in halved worlds. New York: Springer.

Bartolomeo, P., Thiebaut de Schotten, M., \& Chica, A. B. (2012). Brain networks of visuospatial attention and their disruption in visual neglect. Frontiers in Human Neuroscience, 6:110.

Bellgrove, M.A., Eramudugolla, R., Newman, D.P., Vance, A., \& Mattingley, J.B. (2013). Influence of attentional load on spatial attention in acquired and developmental disorders of attention. Neuropsychologia, 51, 1085-1093.

Bisiach, E., Cappa, S.F., \& Vallar, G. (1983). Guida all'esame neuropsicologico. Milano, Italy: Raffaello Cortina Editore.

Bisiach, E., Ricci, R., Lualdi, M., \& Colombo, M.R. (1998). Perceptual and response bias in unilateral neglect: Two modified versions of the milner landmark task. Brain and Cognition, 37, 369-386.

Bonato, M. (2012). Neglect and extinction depend greatly on task demands: a Review. Frontiers in Human Neuroscience, 6:195.

Bonato, M. (2015). Unveiling residual, spontaneous recovery from subtle hemispatial neglect three years after stroke. Frontiers in Human Neuroscience 9:413. doi:10.3389/fnhum.2015.00413

Bonato, M., Priftis, K., Marenzi, R., Umiltà, C., \& Zorzi, M. (2010). Increased attentional demands impair contralesional space awareness following stroke. Neuropsychologia, 48, 3934-3940.

Bonato, M., Priftis, K., Marenzi, R., Umiltà, C., \& Zorzi, M. (2012). Deficits of contralesional awareness: A case study on what paper-and-pencil tests neglect. Neuropsychology, 26, 20-36.

Bonato, M., Priftis, K., Umiltà C., \& Zorzi, M. (2013). Computer-based testing unveils severe neglect in apparently intact patients. Behavioural Neurology, 25, 1-3.

Brozzoli, C., Demattè, M.L., Pavani, F., Frassinetti, F., \& Farnè, A. (2006) Neglect and extinction: within and between sensory modalities. Restorative Neurology and Neuroscience, 24, 217-232.

Buxbaum, L.J., Dawson, A.M., \& Linsley, D. (2012). Reliability and validity of the Virtual Reality Lateralized Attention Test in assessing hemispatial neglect in right-hemisphere stroke. Neuropsychology, 26, 430-441.

Dawson, A., Buxbaum, L.J., \& Rizzo, A.A. (2008). The virtual reality lateralized attention test: Sensitivity and validity of a new clinical tool for assessing hemispatial neglect. IEEE XPlore, Virtual Rehabilitation 88-92. doi:10.1109/ICVR.2008.4625140

Dehaene, S., Kerszberg, M., \& Changeux J. P. (1998). A neuronal model of a global workspace in effortful cognitive tasks. Proceedings of National Academy of Sciences USA, 95, 14529-14534. 
Driver, J., \& Vuilleumier, P. (2001). Perceptual awareness and its loss in unilateral neglect and extinction. Cognition, 79, 39-88.

Ferber, S., \& Karnath, H.O. (2001). How to assess spatial neglect-line bisection or cancellation tasks? Journal of Clinical and Experimental Neuropsychology, 23, 599-607.

Halligan, P.W., \& Marshall, J.C., (1991). Left neglect for near but not far space in man. Nature, 350, 498-500.

Halligan, P.W., Marshall, J.C., \& Wade, D. (1992). Left on the right: Allochiria in a case of left visuo-spatial neglect. Journal of Neurology, Neurosurgery, and Psychiatry, 55, 717-719.

Jacobs, L. (1980). Visual allesthesia. Neurology, 30, 1059-1063.

Jacobs, S., Brozzoli, C., Hadj-Bouziane, F., Meunier, M., \& Farnè, A. (2011). Studying multisensory processing and its role in the representation of space through pathological and physiological crossmodal extinction. Frontiers in Psychology 6, 2:89. doi: 10.3389/fpsyg.2011.00089. Jones, E. (1907). The precise diagnostic value of allochiria. Brain, 30,490-532.

Karnath, H.-O. (2015). Spatial attention systems in spatial neglect. Neuropsychologia, 75, 61-73.

Kinsbourne, M., \& Warrington, E.K. (1962). A disorder of simultaneous form perception. Brain, 85, 461-486.

Lepore, M., Conson, M., Grossi, D., \& Trojano, L. (2003). On the different mechanisms of spatial transpositions: a case of representational allochiria in clock drawing. Neuropsychologia, 41, 12901295.

Liu, C.C., Veldhuijzen, D.S., Ohara, S., Winberry, J., Greenspan, J.D., \& Lenz, F.A. (2011). Spatial attention to thermal pain stimuli in subjects with visual spatial hemi-neglect: extinction, mislocalization and misidentification of stimulus modality. Pain, 152, 498-506.

Magni, E., Benedetti, G., Bianchetti, A., Rozzini, R., \& Trabucchi, M. (1996). Mini-Mental state examination: a normative study in italian elderly population. European Journal of Neurology 3: 1-5. Maravita, A., Posteraro, L., Husain, M., Vuilleumier, P., Schwartz, S., \& Driver, J. (2007). Looking at human eyes affects contralesional stimulus processing after right hemispheric stroke. Neurology, 69, 1619-1621.

Marcel, A., Postma, P., Gillmeister, H., Cox, S., Rorden, C., Nimmo-Smith, I. \& Mackintosh, B.J. (2004). Migration and fusion of tactile sensation: premorbid susceptibility to Allochiria, neglect and extinction? Neuropsychologia, 42, 1749-1767.

Marini, F., Chelazzi, L., \& Maravita, A. (2013). The costly filtering of potential distraction: evidence for a supramodal mechanism. Journal of Experimental Psychology: General, 142, 906922.

Marzi, C.A., Smania, N., Martini, M.C., Gambina, G., Tomelleri, G., Palamara, A., Alessandrini, F., \& Prior, M. (1996) Implicit redundant- targets effect in visual extinction Neuropsychologia, 34, 9-22.

Meador, K.J., Allen, M.E., Adams, R.J., \& Loring, D.W. (1991). Allochiria vs Allesthesia. Is There a Misperception? Archives of Neurology, 48, 546-549.

Medina, J., \& Rapp, B. (2008). Phantom tactile sensations modulated by body position. Current Biology, 18, 1937-1942.

Milner, A.D., Harvey, M., \& Pritchard, C.L. (1998). Visual size processing in spatial neglect. Experimental Brain Research, 123, 192-200.

Mondini, S., Mapelli, D., Vestri, A., \& Bisiacchi, P. (2003). Esame Neuropsicologico Breve. Milano, Italy: Raffaello Cortina Editore.

Neppi-Modona, M. (1999). Influence of response modality on awareness of contralesional tactile stimuli in right brain damaged patients. Cortex, 35, 687-699.

Obersteiner, H. (1882). On allochiria. Brain, 4, 153-168.

Ortigue, S., Jabaudon, D., Landis, T., Michel, C. M., Maravita, A., \& Blanke, O. (2005). Preattentive interference between touch and audition: a case study on multisensory alloesthesia. Neuroreport, 16, 865. 
Pavani, F., Husain, M., Ládavas, E., \& Driver, J. (2004). Auditory deficits in visuospatial neglect patients. Cortex, 40, 347-365.

Peers, P.V., Cusack, R., \& Duncan J. (2006). Modulation of spatial bias in the dual task paradigm: evidence from patients with unilateral parietal lesions and controls. Neuropsychologia, 44, 13251335.

Rees, G., Wojciulik, E., Clarke, K., Husain, M., Frith, C., \& Driver, J. (2000). Unconscious activation of visual cortex in the damaged right hemisphere of a parietal patient with extinction. Brain, 123, 1624-1633.

Robertson, I.H., Manly, T., Andrade, J., Baddeley, B.T., \& Yiend, J. (1997). 'Oops!': performance correlates of everyday attentional failures in traumatic brain injured and normal subjects. Neuropsychologia, 35, 747-758.

Robertson I.H., \& -Frasca R. (1992). Attentional load and visual neglect. International Journal Neuroscience, 62, 45-56.

Robertson, L.C. (2003). Binding, spatial attention and perceptual awareness. Nature Reviews Neuroscience, 4, 93-102.

Ronchi, R., Posteraro, L., Fortis, P., Bricolo, E., \& Vallar, G. (2009). Perseveration in left spatial neglect: drawing and cancellation tasks. Cortex, 45, 300-312.

Russell, C., Husain, M., \& Malhotra, P. (2004). Attention modulates the visual field in healthy observers and parietal patients. Neuroreport, 15, 2189-2193.

Russell, C., Malhotra, P., Deidda, C., \& Husain, M., (2013). Dynamic attentional modulation of vision across space and time after right hemisphere stroke and in ageing. Cortex, 49, 1874-1883.

Sagiv, N. \& Robertson, L.C. (2005). Synesthesia and the Binding Problem. In L.C. Robertson \& N. Sagiv (Eds.), Synesthesia: Perspectives from Cognitive Neuroscience (pp.90-107). New York: Oxford University Press.

Sarri, M., Greenwood, R., Kalra, L., \& Driver, J. (2009). Task-related modulation of visual neglect in cancellation tasks. Neuropsychologia, 47, 91-103.

Schneider, W., Eschman, A., \& Zuccolotto, A. (2002). E-Prime User's Guide. Pittsburgh: Psychology Software Tools, Inc.

Spillane, J. (1938). Olfactory alloesthesia. Brain, 61, 393-401.

Treccani, B., Cubelli, R., Sellaro, R., Umiltà, C., \& Della Sala, S. (2012). Dissociation between awareness and spatial coding: evidence from unilateral neglect. Journal of Cognitive Neuroscience, 24, 854-867.

Treisman, A., \& Schmidt, H. (1982). Illusory conjunctions in the perception of objects. Cognitive Psychology, 14, 107-141.

Treisman, A. (1996). The binding problem. Current Opinion Neurobiology, 6, 171-178.

Treisman, A. (1998). Feature binding, attention and object perception. Philosophical Transactions of the Royal Society of London: Series B, Biological Sciences, 353, 1295-1306.

van Kessel, M.E., van Nes, I.J., Geurts, A.C., Brouwer, W.H., \& Fasotti, L. (2013). Visuospatial asymmetry in dual-task performance after subacute stroke. Journal of Neuropsychology, 7, 72-90.

Vuilleumier, P. (2013). Mapping the functional neuroanatomy of spatial neglect and human parietal lobe functions: progress and challenges. Annals New York Academy Science, 1296, 50-74.

Vuilleumier, P., Schwartz, S., Verdon, V., Maravita, A., Hutton, C., Husain, M., \& Driver, J. (2008). Abnormal attentional modulation of retinotopic cortex in parietal patients with spatial neglect. Current Biology, 18, 1525-1529.

Wilson, B., Cockburn, J., \& Halligan, P. W. (1987). The behavioural inattention test. Bury St. Edmunds, UK: Thames Valley Test Company. 
Table 1 Sample characteristics and neuropsychological profiling

\begin{tabular}{|c|c|c|c|c|c|c|c|c|c|c|c|c|c|c|}
\hline $\begin{array}{l}\text { Patient } \\
\text { number }\end{array}$ & $\begin{array}{l}\text { Age } \\
\text { (yrs) }\end{array}$ & Sex & $\begin{array}{l}\text { Education } \\
\text { (yrs) }\end{array}$ & $\begin{array}{l}\text { Days } \\
\text { from } \\
\text { onset }\end{array}$ & MMSE & $\begin{array}{c}\text { Lesion } \\
\text { Ethiolo } \\
\text { gy }\end{array}$ & $\begin{array}{l}\text { Lesion } \\
\text { site }\end{array}$ & BIT & $\begin{array}{c}\text { Lines } \\
\mathrm{L}\end{array}$ & $\begin{array}{c}\text { Lines } \\
\mathrm{R}\end{array}$ & $\begin{array}{l}\text { Letters } \\
\text { L }\end{array}$ & $\begin{array}{c}\text { Letters } \\
\mathrm{R}\end{array}$ & $\begin{array}{c}\text { Stars } \\
\mathrm{L}\end{array}$ & $\begin{array}{c}\text { Stars } \\
\mathrm{R}\end{array}$ \\
\hline 1 & 78 & $\mathbf{F}$ & 4 & 476 & $22(21)$ & Is & $\begin{array}{c}\text { T, P, } \\
\text { SC }\end{array}$ & 134 & 18 & 18 & 20 & 17 & 20 & 25 \\
\hline 2 & 85 & M & 13 & 40 & $26.3(26)$ & $\mathrm{He}$ & $\begin{array}{l}F, P, \\
\text { SC }\end{array}$ & 131 & 18 & 18 & 17 & 19 & 26 & 26 \\
\hline 3 & 64 & $\mathbf{F}$ & 5 & 34 & 26 & Is & $\begin{array}{c}F, I, P, \\
\text { SC }\end{array}$ & 138 & 18 & 18 & 20 & 15 & 27 & 27 \\
\hline 4 & 77 & M & 5 & 76 & $25.7(26)$ & Is & $\begin{array}{c}F, I, T, \\
\text { SC }\end{array}$ & 124 & 18 & 18 & 9 & 13 & 26 & 27 \\
\hline 5 & 73 & $\mathbf{F}$ & 5 & 61 & $27.3(28)$ & $\mathrm{He}$ & SC, C & 135 & 18 & 18 & 20 & 18 & 22 & 24 \\
\hline 6 & 61 & M & 13 & 31 & 28 & Is & I, T, SC & 145 & 18 & 18 & 20 & 19 & 27 & 27 \\
\hline 7 & 53 & M & 8 & 75 & 27 & $\mathrm{He}$ & SC & 131 & 18 & 18 & 17 & 18 & 24 & 27 \\
\hline 8 & 40 & $\mathbf{F}$ & 8 & 47 & 28 & $\mathrm{Ca}$ & P, SC & 129 & 18 & 18 & 17 & 17 & 26 & 23 \\
\hline 9 & 74 & $\mathbf{F}$ & 2 & 24 & 19.7 (19) & $\mathrm{He}$ & SC & 136 & 18 & 18 & 15 & 19 & 25 & 26 \\
\hline
\end{tabular}

Lesion site:

$\mathrm{F}=$ frontal $; \mathrm{P}=$ parietal $; \mathrm{T}=$ temporal $; \mathrm{I}=$ insula $; \mathrm{C}=$ capsular; $\mathrm{SC}=$ Subcortical

MMSE: For patients aged 65 or more, raw scores (between brackets) have been corrected according to Magni et al. (1996).

Etiology:

Is $=$ ischemic; $\mathrm{He}=$ hemorrhagic.

BIT: For each cancellation subtest (Lines, letters, stars), the number of detected items is shown, separately for the left (L) and the right (R) half of the testing sheet. Scores $\leq 129$ are to be considered pathological.

Table 2: Response percentage according to target side and type of task.

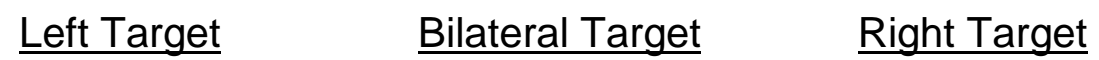

\begin{tabular}{|c|c|c|c|c|c|c|c|c|c|c|c|c|c|}
\hline \multirow{3}{*}{$\begin{array}{c}\text { Patient } \\
\text { number } \\
1\end{array}$} & \multirow{3}{*}{$\begin{array}{c}\text { Task } \\
\text { ST }\end{array}$} & & \multicolumn{11}{|c|}{ Response (\%) } \\
\hline & & $0^{n}$ & Left & Bilat $^{s}$ & $\operatorname{Right}^{a}$ & 0 & Left & Bilat & Right $^{e}$ & 0 & Left & Bilat & Right \\
\hline & & 4 & 92 & 4 & 0 & 0 & 0 & 93.8 & 6.3 & 0 & 0 & 0 & 100 \\
\hline & VDT & 0 & 41.7 & 54.2 & 4.2 & 0 & 0 & 96.3 & 3.7 & 0 & 0 & 16 & 84 \\
\hline & ADT & 0 & 96.3 & 3.7 & 0 & 0 & 6.7 & 83.3 & 10 & 0 & 3.4 & 0 & 96.6 \\
\hline 2 & ST & 54.8 & 45.2 & 0 & 0 & 0 & 9.7 & 6.5 & 83.9 & 0 & 0 & 0 & 100 \\
\hline & VDT & $14.8^{\wedge}$ & 18.5 & 0 & 66.7 & 0 & 0 & 0 & 100 & 0 & 0 & 0 & 100 \\
\hline & ADT & $20^{*}$ & 70 & 0 & 10 & 3.2 & 0 & 3.2 & 93.5 & 3.2 & 0 & 0 & 96.8 \\
\hline 3 & ST & 9.4 & 90.6 & 0 & 0 & 3.1 & 0 & 68.8 & 28.1 & 0 & 0 & 0 & 100 \\
\hline & VDT & $90.3^{*}$ & 0 & 0 & 9.7 & 0 & 0 & 0 & $100^{*}$ & 0 & 0 & 0 & 100 \\
\hline & ADT & $90.6^{*}$ & 0 & 0 & 9.4 & 0 & 0 & 0 & $100^{*}$ & 0 & 0 & 0 & 100 \\
\hline
\end{tabular}




\begin{tabular}{|c|c|c|c|c|c|c|c|c|c|c|c|c|c|}
\hline \multirow[t]{3}{*}{4} & ST & 90.6 & 6.3 & 0 & 3.1 & 0 & 0 & 0 & 100 & 0 & 0 & 0 & 100 \\
\hline & VDT & $35^{\wedge}$ & 0 & 0 & 65 & 4.5 & 0 & 0 & 95.5 & 0 & 0 & 0 & 100 \\
\hline & ADT & $65.6^{\wedge}$ & 0 & 0 & 34.4 & 6.3 & 0 & 0 & 93.8 & 0 & 0 & 0 & 100 \\
\hline \multirow[t]{3}{*}{5} & ST & 9.7 & 90.3 & 0 & 0 & 9.4 & 0 & 87.5 & 3.1 & 0 & 0 & 0 & 100 \\
\hline & VDT & $35.7^{*}$ & 60.7 & 3.6 & 0 & 0 & 3.4 & 48.3 & $48.3^{*}$ & 0 & 0 & 4 & 96 \\
\hline & ADT & $37.5^{*}$ & 50 & 0 & 12.5 & 0 & 0 & 48.4 & $51.6^{*}$ & 3.2 & 0 & 0 & 96.8 \\
\hline \multirow[t]{3}{*}{6} & ST & 0 & 100 & 0 & 0 & 0 & 0 & 93.8 & 6.3 & 0 & 0 & 0 & 100 \\
\hline & VDT & 6.5 & 93.5 & 0 & 0 & 0 & 0 & 18.8 & $81.3^{*}$ & 0 & 0 & 0 & 100 \\
\hline & ADT & $34.5^{*}$ & 65.5 & 0 & 0 & 0 & 0 & 19.4 & $80.6^{*}$ & 0 & 0 & 0 & 100 \\
\hline \multirow[t]{3}{*}{7} & ST & 84.4 & 12.5 & 0 & 3.1 & 0 & 0 & 6.3 & 93.8 & 6.3 & 0 & 0 & 93.8 \\
\hline & VDT & 95.2 & 0 & 0 & 4.8 & 8 & 0 & 0 & 92 & 5 & 0 & 0 & 95 \\
\hline & ADT & 84.4 & 3.1 & 0 & 12.5 & 3.3 & 0 & 0 & 96.7 & 6.9 & 0 & 0 & 93.1 \\
\hline \multirow[t]{3}{*}{8} & ST & 90.6 & 0 & 0 & 9.4 & 0 & 0 & 6.3 & 93.8 & 6.3 & 0 & 3.1 & 90.6 \\
\hline & VDT & 90.6 & 6.3 & 0 & 3.1 & 0 & 0 & 0 & 100 & 3.4 & 0 & 0 & 96.6 \\
\hline & ADT & 71.9 & 0 & 0 & 28.1 & 3.1 & 0 & 0 & 96.9 & 6.3 & 0 & 0 & 93.8 \\
\hline \multirow[t]{3}{*}{9} & ST & 33.3 & 66.7 & 0 & 0 & 9.4 & 0 & 62.5 & 28.1 & 6.3 & 3.1 & 0 & 90.6 \\
\hline & VDT & $100^{*}$ & 0 & 0 & 0 & 57.1 & 0 & 0 & $42.9^{*}$ & 30.8 & 0 & 0 & 69.2 \\
\hline & ADT & $87.5^{*}$ & 9.4 & 0 & 3.1 & 18.8 & 0 & 0 & $81.3^{*}$ & 25 & 0 & 0 & 75 \\
\hline
\end{tabular}

ST: Single-Task

VDT: Visual Dual-Task

ADT: Auditory Dual-Task

${ }^{\mathrm{n}}=$ neglect,${ }^{\mathrm{s}}=$ synchiria, ${ }^{\mathrm{a}}=$ alloesthesia,${ }^{\mathrm{e}}=$ extinction

Bold: correct response

Shaded, light grey: synchiria $=$ significant increase in "bilateral" responses to left targets with respect to $\mathrm{ST}$

Shaded, dark grey: alloesthesia = significant increase in "right" responses to left targets with respect to ST

* = significant increase in omissions (unilateral left targets) or extinction (bilateral targets) with respect to $S T$

$\wedge=$ significant decrease in omissions (unilateral left targets) with respect to ST 